\title{
Monte Carlo Methodology for Grand Canonical Simulations of Vacancies at Crystalline Defects
}

\author{
Döme Tanguy \\ CNRS, UMR 5146, Ecole des Mines de Saint-Etienne
}

France

\section{Introduction}

The design of new materials and the optimization of the existing ones require more and more knowledge of the elementary processes underlying the macroscopic properties. Computer simulations have become, together with ever finer experimental technics, the modern tools for probing these mechanisms. This paper focuses on the development of Monte Carlo simulations, at the atomic scale, of vacancies in crystals. These defects have been extensively studied, in their isolated state, because they are the vectors of diffusion in solids. Their concentration and dynamics determine the kinetics of most phase transformations and thermal annealings which enter the processes of the production of materials, for example metallic alloys, or surface deposits for microelectronic applications. They can also contribute to the loss of mechanical properties. For example, in irradiated steels, the clustering of vacancies induce the formation of loops which harden the matrix and, at the same time, their diffusion to the grain boundaries lead to all sorts of segregations that sometimes reduce their cohesion. The combined effect of a hard matrix and weak interfaces can lead to the premature formation of cracks. It is therefore not only important to model vacancies in a hole range of temperatures and concentrations in perfect crystals, but also at pre-existing defects, like grain boundaries and dislocations.

The methodology presented is inherited from statistical mechanics. Molecular Dynamics (MD) (Allen \& Tildesley (1991)) is the method of choice if the details of the trajectories of the particles in the system are needed. The amount of physical time that can be simulated (of the order of the nano second) is often too limited to give enough statistics to measure the property of interest. Kinetic Monte Carlo (Landau \& Binder (2000); Soisson et al. (1996); Dai et al. (2005)) is event based. It eliminates all the details of the trajectory and keeps only the jumps from one local minium of the energy to another one. In its simple form, a limited list of the most important events is provided at the beginning of the simulation, together with the list of rates and the particles are constrained to be on a rigid lattice. It can be refined to build the list on the fly (Henkelmann \& Jónsson (2001)) or to get the events from MD (Sørensen \& Voter (2000)). A last class of methods is the one where MD is accelerated (Voter et al. (2001)), for example, by the use of a bias in the interactions (Wang et al. (2001)) which does not modify the saddles in between the local energy minima, but reduces the waiting time in the basins. Each method has its limitations: KMC, on a rigid lattice, can not treat realistic diffusion mechanisms with collective movements and relaxations (for example, if the system has different components 
with marked different sizes), Accelerated Dynamics is sometimes still limited to short time scales when many low barriers are present.

We feel the need to develop some tools which lie in between rigid lattice methods and MD by borrowing some of their characteristics. First of all, this study is focused on simulating equilibrium configurations, but in rather complex geometries like grain boundaries. Monte Carlo and Molecular Dynamics are used, together, to treat interstitial-vacancy clusters and vacancies at grain boundaries. This effort can only be considered as a first step since, most of the time, out of equilibrium situations are met in experiments. It means that the work should be oriented towards rate calculations in the future.

The paper is organized as follows: A review of equilibrium Monte Carlo simulations in the Grand Canonical ensemble is given. Insertion/deletions moves are presented. They are used to obtain a fluctuating number of particles in the system. The biases that have been developed to extend this method to dense systems are also presented. Next, we detail our model which is an intermediate between a rigid lattice and a continuum model. An application to the simulation of thermal vacancies at a grain boundary is given. Then the model is enriched to treat also interstitial solutes. Vacancy-hydrogen clusters are simulated in a perfect crystal. The focus is on the design of cluster moves. The method is extended to grain boundaries. $\mathrm{H}$ segregation is shown as an example. The problem of the slow convergence is discussed in the case of vacancy-hydrogen co-segregation. Finally, an algorithm is developed to solve this problem and is applied to the ordering of vacancies alone in a grain boundary.

\section{Simulations in the Grand Canonical ensemble, in dense systems}

The method for simulating the Grand Canonical ensemble is briefly reviewed, first in the context of the low density systems such as gazes or low density fluids. Next, the modifications that were made to extend this method to dense liquids or the hexatic phase are presented.

Consider a set of $\mathrm{N}$ labeled classical particles, in a volume $\mathrm{V}$ at temperature $\mathrm{T}$. A microstate of the system is characterized by the continuous positions and momenta of the particles: $\left\{q_{i}\right\}^{N}$ $\left\{p_{i}\right\}^{N}$, where $q$ and $p$ are vectors and $i$ is the label of the particle. One microstate is associated to an infinitesimal volume of the space $\left(\left\{q_{i}\right\}^{N},\left\{p_{i}\right\}^{N}\right)$, proportional to $\left(\left\{d q_{i}\right\}^{N},\left\{d p_{i}\right\}^{N}\right)$, the uncertainty of the measure of the positions and impulsions. Consider that this system is in equilibrium with a reservoir with which it can exchange particles, such that the chemical potential is fixed (all particles are considered to be of the same chemical nature). The probability that the system be in the microstate $\left(N,\left\{q_{i}\right\}^{N},\left\{p_{i}\right\}^{N}\right)$ is:

$$
\begin{array}{r}
p\left(N,\left\{q_{i}\right\}^{N},\left\{p_{i}\right\}^{N}\right)\left\{d q_{i}\right\}^{N}\left\{d p_{i}\right\}^{N}=\frac{1}{\mathcal{Q}(\mu, V, T)} \frac{1}{N !} \\
\exp \left(-\beta\left(H\left(\left\{q_{i}\right\}^{N}\right)+\frac{1}{2} \sum_{i=1}^{N} 1 / 2 m_{i} p_{i}^{2}-N \mu\right)\right) \frac{\left\{d q_{i}\right\}^{N}\left\{d p_{i}\right\}^{N}}{h^{3 N}}
\end{array}
$$

where $\mathcal{Q}(\mu, V, T)$ is the partition function:

$$
\mathcal{Q}(\mu, V, T)=\sum_{N=0}^{+\infty} \int \frac{1}{h^{3 N} N !} \exp \left(-\beta\left(H\left(\left\{q_{i}\right\}^{N}\right)+\frac{1}{2} \sum_{i=1}^{N} \frac{1}{2 m_{i}} p_{i}^{2}-N \mu\right)\right)\left\{d q_{i}\right\}^{N}\left\{d p_{i}\right\}^{N}
$$


In the case where the quantities of interest do not explicitely depend on the velocities of the particles, only the configurational part of the density can be considered and the kinetic part is integrated by hand. Equation 1 becomes:

$$
p\left(N,\left\{q_{i}\right\}^{N}\right)\left\{d q_{i}\right\}^{N}=\frac{1}{\mathcal{Q}(\mu, V, T)} \frac{1}{N ! \Lambda^{3 N}} \exp \left(-\beta\left(H\left(\left\{q_{i}\right\}^{N}\right)-N \mu\right)\right)\left\{d q_{i}\right\}^{N}
$$

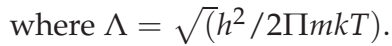

In order to let the number of particles fluctuate (since the extensive variable $\mathrm{V}$ is fixed and that the particles cannot overlap strongly, the number of particles oscillates around an average value), Monte Carlo simulations can be performed with a specific trial move: the insertion/deletion move.

The probability of the transition from a state o (old) to a state $\mathrm{n}$ (new) is decomposed: $p_{o \rightarrow n}=\rho_{o} \alpha_{o \rightarrow n} a c c_{o \rightarrow n}$, with $\rho_{o}$ the probability that the system, in equilibrium, is in state o (Eq. 3); $\alpha$ is the probability to propose the transition (trial) and acc is the probability to accept the transition. Let's consider that the $\mathrm{n}$ state is obtained by inserting, at random, a particle in volume $\mathrm{V}$. The probability to pick the new position $q_{N+1}$ within the box of length $d q_{N+1}$ is $d q_{N+1} / V$, if we imagine that physical space is decomposed in small boxes of length $d q$. The position of all the other particles is left unchanged. Once the new particle is inserted, the particles need to be relabeled. We take one label at random between 1 and $(\mathrm{N}+1)$ for the new particle and we proceed like that for all particles sequentially. The new labeling is obtained with probability $1 /(\mathrm{N}+1)$ !. The reverse move is obtained by selecting the former "new" particle among the others. This is done with probability $1 /(\mathrm{N}+1)$. The particle is removed and the configuration is relabeled. This gives N! possibilities. The probability to come back to the old configuration, with the same labeling is $1 / \mathrm{N}$ !. A sufficient condition to ensure that the Markov chain constructed by proposing insertion/deletions produces the desired equilibrium distribution of states (coherent with $\rho$ eq. 3 ) is "detailed balance":

$$
\rho_{o} \alpha_{o \rightarrow n} a c c_{o \rightarrow n}=\rho_{n} \alpha_{n \rightarrow o} a c c_{n \rightarrow o}
$$

where $\alpha_{o \rightarrow n}=d q_{N+1} / V \times 1 /(N+1)$ ! and $\alpha_{n \rightarrow 0}=1 /(N+1) \times 1 / N$ !. Substituting Eq. 3 and the values of $\alpha$ discussed in the text gives:

$$
\begin{gathered}
\frac{1}{\Lambda^{3 N} N ! \mathcal{Q}} \exp \left(-\beta\left(H\left(\left\{q_{i}\right\}^{N}\right)-N \mu\right)\right) d q_{i}^{N} \frac{d q_{N+1}}{V(N+1) !} a c c_{0 \rightarrow n}=\frac{1}{\Lambda^{3(N+1)}(N+1) ! \mathcal{Q}} \\
\exp \left(-\beta\left(H\left(\left\{q_{i}\right\}^{N}, q_{N+1}\right)-(N+1) \mu\right)\right) d q_{i}{ }^{N} d q_{N+1} \frac{1}{(N+1) N !} a c c_{n \rightarrow 0} \\
\frac{a c c_{o \rightarrow n}}{a c c_{n \rightarrow 0}}=\frac{V}{(N+1) \Lambda^{3}} \exp \left(-\beta\left(H\left(\left\{q_{i}\right\}^{N}, q_{N+1}\right)-H\left(\left\{q_{i}\right\}^{N}\right)-\mu\right)\right)
\end{gathered}
$$

The new configuration is accepted according to the Metropolis rule (Allen \& Tildesley (1991)). This algorithm has been used to simulate the equilibrium between a gas and a liquid (Adams (1975); Rowley et al. (1976)), generalized to Coulombic systems (Valleau \& Cohen (1980)) and also applied to electrical double layers (van Megen \& Snook (1980)).

The critical part is the random insertion. When the density becomes large, the probability that the trial position gives a large overlap with an existing particle increases. The energy variation becomes large and the acceptance ratio drops. The sampling becomes inefficient. Mezei (Mezei (1980)) proposed to bias the insertions by detecting cavities in the system and extended the simulations to dense liquids. These ideas where revisited and extended by Swope (Swope \& 
Andersen (1992; 1996)). His algorithm is briefly presented. An interesting use of a grid is made to design an efficient insertion/deletion move, in the spirit of Mezei.

The idea is to attempt an insertion only at a position where it has a high probability to be accepted, respectively attempt a deletion of a particle that has a non negligible chance to be successful. This choice depends on the "old" configuration and also on the "new" configuration, because of the detailed balance condition. In order to construct the move, the volume of the system is decomposed in identical small cubic cells (the size is a fraction of the nearest neighbor distance). Their center is also considered. This decomposition is made at the beginning of the simulation and is not modified. The most relevant cells are extracted and called ID cells (ID: insertion deletion). The metropolis criterion contains the number of filled ID cells and the number of empty ID cells in addition to the usual energy variation term. There is a lot of flexibility in the definition of an ID cell. Swope chose, for the example he treated, to proceed in two steps:

- A geometric criterion: an ID cell, independently of whether it is occupied or not, has no particles closer than a fixed radius to its center. This is to avoid strong overlaps and to limit the number of ID cells in a computationally cheap way.

- An energy criterion: in the cells that satisfy the geometric criterion, a particle is inserted at their center (a fixed position, to satisfy reversibility) and the energy variation $\Delta H$ is calculated (if a cell is occupied, the contribution of the particle is not considered). The cell is considered an ID cell if $\Delta H$ is in a narrow range, which is common to the insertion and the deletion.

This energy range is chosen to increase the acceptance rate. Once the ID cells are defined and selected for the move, the insertion is performed at random in the ID cell if it is empty. Otherwise, the particle is removed. For the fluid that was studied, modeled by a twelfth power repulsive interaction, $20 \%$ of acceptance could be reached, which is remarkably high.

This algorithm uses a lattice (the cell centers), even if the system is not necessarily crystalline, which is original. The energy criterion, on the other hand, is quite expensive since it requires evaluation of many variations of energies.

\section{Dealing with vacancies}

Defining a vacancy implies that some degree of order, even only local or metastable, exists. A lattice structure can be defined by averaging the positions of the particles over a time scale smaller that the typical time scale for the disappearing of this local order. A vacancy is then an unoccupied lattice site. If, in simple crystalline structures, like fcc or bcc, the vacancies are usually well localized, it is not necessarily the case when crystalline defects are present. A spectacular example is given in (Denkowicz et al. (2008)). Atomic scale simulations of $\mathrm{Cu}-\mathrm{Nb}$ multilayer systems, with layers being $4 \mathrm{~nm}$ thick, were conducted. The $\mathrm{Cu}-\mathrm{Nb}$ interfaces have several dense arrays of misfit dislocations. When introduced, vacancies delocalize and trigger complex dislocation rearrangements. As a consequence, the interface energy can be reduced by incorporating up to $5 \%$ of vacancies (the reference structure is the one obtained by sticking perfect layers, with different misorientations). This is a particular example where, by construction, the initial structure is stressed. In the case of a grain boundary, the system is trapped in a metastable state by the application of macroscopic constraints: the misorientation and the relative translation of the constitutive crystals. When looking for the ground state, atoms are removed until the lowest excess energy is found. It is common that several arrangements have close energies so that several structures coexist at finite temperature. 
$\Sigma 5(210)[001]$ symmetrical tilt boundary has this property and can oscillate between two structures by migration of the interface (shear and translation perpendicular to the GB plane) or by absorption/emission of a vacancy per structural unit. It is therefore not straightforward to define a lattice for the GB core structure that can be suited to define vacancies. Furthermore, the localized state is not the only one for the vacancy: delocalization-re localizations are frequent during diffusion events. They can be simple exchanges with first neighbors or complex collective moves (Sørensen et al. (2000)), see (Suzuki \& Mishin (2005)) for a review. Furthermore, when simulating crystalline systems, the initial size and shape of the box, which typically contains an integer number of elementary cells, imposes a strong constraint on the number of lattice sites which is not compatible with the equilibrium concentration of vacancies. These constraints, discussed by Swope (Swope \& Andersen (1992)), can affect drastically the results of simulations of transitions between phases of very different structures (for example: melting (Bagchi et al. (1996))). In our case, large concentrations of vacancies, with a large number of lattice sites are involved (for a reason explained later). The impact of the constraint on the average concentrations of vacancies are not dramatic. Nevertheless, the issue of the unphysical distortions, also discussed in Swope's papers, are relevant and we do check the structures for them.

We use a model which is intermediate between a rigid lattice (Ising) and continuum. The particles are no longer represented by their positions respective to an origin but by a displacement relative to a lattice node (Tanguy \& Mareschal (2005)). Node occupations (analogous to the spins of the Ising model) are defined: $p_{i}=1$ if node number $i$ is occupied by a particle, $p_{i}=0$ is it is empty (vacancy). Furthermore, the displacements are confined to the Voronoï cells around the nodes. This constraint is natural in solids. No self interstitials are allowed. This is also acceptable if the crystal is stable and in equilibrium. A similar model was used for simulating the phase diagram of bulk Si-Ge, using a diamond lattice (Dünweg \& Landau (1993)). Although the authors present their model as being able to handle vacancies, the insertion/deletion is not presented and the calculations were done, in practice in the semi-grand canonical ensemble.

With these considerations, a microstate is defined by the set of the occupancies and the displacements, when the nodes are filled: $\left(\left\{p_{i}\right\}^{M},\left\{\vec{u}_{i}\right\}^{N}\right)$. There is one extensive variable in addition to the traditional ones which is the number of nodes $\mathrm{M}$ ( $\mathrm{N}$ is the number of particles). M can be used to free the volume as discussed in (Tanguy \& Mareschal (2005)). The partition function is:

$$
\begin{array}{r}
\mathcal{Q}_{c}(M, \mu, V, T)=\sum_{N=0}^{M} \sum_{\left\{p_{n}\right\}} \frac{1}{\Lambda^{3 N}} \int_{W . S} d \vec{u}^{N} \\
\times \exp \left(-\beta\left(\mathcal{H}\left(\left\{p_{n}\right\},(\vec{u})^{N}\right)-N \mu\right)\right)
\end{array}
$$

where $\mu$ is the chemical potential. The second sum (over the set of occupancy numbers) represents all the possible arrangements of the vacancies on the nodes of the lattice. Note that the integration of the displacement is over the volume of the Wigner-Seitz cell W.S. Monte Carlo simulations are done according to the density defined by Eq. 7 by:

- Proposing displacements to the particles, within their cell. If a random displacement increment brings the particle out of its cell, the new cell is identified. If it is empty, the occupancies are exchanged. If it is occupied, the move is considered as an attempt to have a double occupancy, which is not possible. A warning is issued and the move is abandoned. 
In practice, it never happens since $\mathrm{T}$ is not close to the melting point. Nevertheless, the possibility is discussed below.

- The number of particles fluctuates. Insertion/deletions are proposed. First a node is selected at random. If it is occupied, the particle is deleted (the occupancy is set to 0). If it is not occupied, a random position is selected in the cell and the particle is inserted. Detailed balance constrains the acceptance in a similar way to Eq. 6:

$$
\frac{a c_{A \rightarrow B}}{\operatorname{acc}_{B \rightarrow A}}=\exp \left(-\beta\left(\mathcal{H}_{B}-\mathcal{H}_{A}+\mu\right)\right) \frac{\Lambda^{3}}{W . S}
$$

where $A$ is the initial state with $\mathrm{N}$ particles and B is the trial state with $\mathrm{N}-1$ particles.

- Exchanges are also proposed between a vacancy and a particle. If the cells have exactly the same shape (which is the case in the perfect lattice, but not in the grain boundary), the displacement of the particle is preserved. The exchanges are necessary to speed up the ordering of the vacancies.

This model is interesting because:

- It provides a simple and unambiguous definition of vacancies.

- Relaxations due to the presence of crystalline defects are taken into account (the displacement moves lead to the relaxation of the defects).

- The cell decomposition of space is used as insertion/deletion cells in the spirit of Swope.

- The lattice can be used to design cluster moves that speed up convergence.

- Lattice models, in the mean field approximation, can be used to check convergence in the low vacancy concentration limit.

The model is used in (Vamvakopoulos \& Tanguy (2009)) to simulate thermal vacancies, at high temperatures, in the $\Sigma 33(554)$ [110] symmetrical tilt boundary (Fig. 1). Because of the displacements, the equilibrium vacancy concentrations take into account the vibrational entropy which largely influences the formation energies in the different sites of the grain boundary. The Monte Carlo results are compared to free energy calculations using the Widom insertion method, which enabled to check the convergence. Clusters of vacancies, up to 5 vacancies along the tilt axis, are observed. Nevertheless the convergence is difficult because of the strong relaxations around the vacancies in the grain boundary. A solution for this problem is presented in the section concerning "Hybrid Monte Carlo".

It is natural to question the motivation for ignoring the self-interstitials in the case of the grain boundary. First, the systematic study of grain boundary diffusion (Suzuki \& Mishin (2005)), show that in high energy boundaries, vacancies and self interstitials have similar formation energies and therefore, in equilibrium, similar concentrations. Furthermore, they are both involved in diffusion at low temperature. We have done the choice to focus on vacancies because we plan to work with large concentrations of vacancies, at low temperature. In this case, the influence of the low concentration of equilibrium self-interstitials is neglected. Note that large distorsions are supposed to be handled by the lattice as it is, i.e. by the combination of a vacancy and the distortions of the neighbors within the limit of their cells. If it is not enough (attempts for double occupancies are tracked by the code), interstitial sites should be included. It would induce a refinement of the Voronoï decomposition because these new sites would be added to the regular lattice sites. Because space is re decomposed, the addition of extra sites, with extra occupancies, do not induce redundancies in the way the microstates 

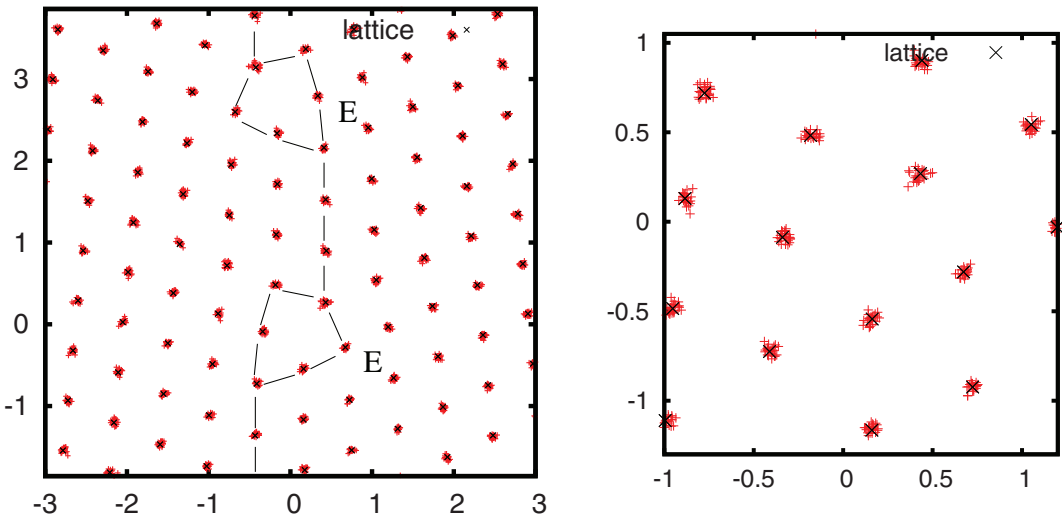

Fig. 1. Projection of the structural unit of the $\Sigma 33(554)$ [110] symmetrical tilt boundary. (x) represents the position of the nodes. (+) are the actual positions of the atoms at thermal equilibrium, without vacancies, at $\mathrm{T}=200 \mathrm{~K}$.

are represented. The new decomposition might not be relevant and, if the original lattice is no longer adapted, it would be easier and more meaningful to use a refined cubic grid such as the one used by Swope, at least in the GB core region. If this is necessary, the concept of vacancy should be abandoned.

\section{Interstitial-vacancy clusters simulations in the Grand Canonical ensemble}

There are different conditions where large concentrations of vacancies can be found. These can be heavily out of equilibrium: irradiation, intense localized plastic deformation (fatigue), oxidation (in particular alloys where one component is preferentially oxidized, the depleted zone is a direct evidence of enhanced diffusion, probably due to large vacancy concentrations). There exist also specific conditions where this can be observed, at equilibrium, in the case of a large binding between the vacancy and the solute introduced in the crystal. It is the case of many metal-hydrogen systems (Fukai (1993); Fukai \& Ōkuma (1994)). In Ni, under high partial pressure of hydrogen and high temperature, the vacancy concentration can be as high as $25 \%$. The origin of the enhanced equilibrium vacancy concentration lies on a significant binding and on the possibility of multiple occupancy of the vacancy by the solute. Indeed, it is known experimentally for a long time (Bugeat et al. (1976)) that $\mathrm{H}$ is not centered in the vacancy. In the eighties, Effective Medium Theory came to a good agreement with experiments and gave a simple and clear picture of $\mathrm{H}$ in metals (for a review see (Nørskov \& Besenbacher (1987))): the minimum potential energy for $\mathrm{H}$ in the vacancy is intermediate between the geometric interstitial position and the center of the vacancy. Multiple occupancy is possible, but the $\mathrm{H}-\mathrm{H}$ interaction in the vacancy is repulsive. This picture has been confirmed since then by ab initio calculations in many metals: Fe (Tateyama \& Ohno (2003)), Al (Gunaydin et al. (2008); Wolverton et al. (2004)), W (Liu et al. (2009)), Be (Ganchenkova et al. (2009))... At zero temperature, multiple occupancy can lead to negative formation energies for the $V H_{n}$ (a vacancy and $\mathrm{n}$ trapped hydrogen). Nevertheless, at finite temperature, the probability that $\mathrm{H}$ is actually in the vacancy depends on the concentration of hydrogen remaining on the regular interstitial sites (the chemical potential of $\mathrm{H}$ ). It is therefore not straightforward 
to conclude, from zero $\mathrm{K}$ binding energies, that $\mathrm{VH}_{n}$ clusters are stable. There can be some misunderstanding with regards to the experimental conditions: if the vacancy is forced into the crystal (by irradiation for example, or by corrosion) and if $\mathrm{H}$ is also present, it will segregate to the vacancy because of its much higher mobility. It might happen that the cluster is much less mobile than the free vacancy and that it gives the impression that the vacancies are stabilized by H (because they don't annihilate within the time scale of the experiment). It does not mean that the clusters are thermodynamically stable.

In the following, the equilibrium Monte Carlo method is extended to simulate the existence domain $\left(C_{H}, \mathrm{~T}\right)$ of vacancy clusters in the conditions where the chemical potential of the metal is imposed (i.e. the crystal is free to eliminate or create vacancies, as if the annealing was lasting for an infinite time in the experiment). The reason why the focus is on equilibrium simulations, and not on kinetic simulations (that would certainly be more relevant to the experimental conditions) is because sampling properly the equilibrium states and getting enough statistics, is already far from being granted, while equilibrium MC gives the advantage to use unphysical moves to get better convergence. This is the theme that is treated now.

\subsection{Grand canonical simulations in a perfect crystal}

In a similar way to what is done for the metal, a lattice is introduced to handle interstitial solutes (Tanguy \& Mareschal (2005)). In the fcc structure, it is composed of one octahedral site and two tetrahedral sites per fcc site. Space is decomposed in Voronoï cells based on this lattice. The interstitials are referenced by an occupation number and a displacement from the node of the cell where they belong. Phase space is extended and sampled by Monte Carlo moves: displacements and exchanges between occupied and empty nodes.

Let's imagine that a vacancy is formed. The exchange moves bring hydrogen in it and an equilibrium is created between the vacancies, the $\mathrm{H}$ which multiply occupy them and the remaining $\mathrm{H}$ on bulk interstitial sites. Suppose an exchange move is attempted for a vacancy containing $\mathrm{n} \mathrm{H}$. After the particle is brought on the empty site, the trial state will have one more free vacancy and $\mathrm{n}$ more isolated $\mathrm{H}$ on bulk sites. The energy variation would be of the order of $n \times E_{b}$ where $E_{b}$ is the binding energy. For $\mathrm{H}$ in $\mathrm{Al}, E_{b} \approx 0.3 \mathrm{eV}$, which, if $n=3$, gives $\Delta E=0.9 \mathrm{eV}$ and the probability to accept the move (metropolis) is $\exp (-0.9 / k T) \approx 10^{-15}$ at $\mathrm{T}=300 \mathrm{~K}$. The direct exchange is never accepted. Not only the ordering of the vacancies is not described, but also the average occupancy (average $\mathrm{n}$ per vacancy) is not correct because vacancy clusters $V_{2} \ldots V_{m}$ do not trap $\mathrm{H}$ in the same way as an isolated vacancy.

A solution is to perform "cluster moves" i.e. to exchange a vacancy and the H atoms it contains, at the same time. Before we do this, we have to come back to the way the microstates are defined. For symmetry reasons, the tetrahedral sites coincide with the corners of the Voronoï polyhedron (and the octahedral site sits on an edge). It means that two vacancies in first neighbor position share 2 tetrahedral sites. If they were occupied, the $\mathrm{H}$ would relax towards the center of the vacancy and therefore, a T site can be occupied by $2 \mathrm{H}$ atoms if each of them relaxes towards a different vacancy. It is possible that not allowing multiple occupancy prevents physical states to appear in the simulation. For this reason we decided to "split" all the interstitial sites. To each fcc site is associated $8 \mathrm{~T}$ and $6 \mathrm{O}$ sites. Each of them is degenerate: the same $\mathrm{T}$ site appears 4 times but associated to four different atoms (the corners of the tetrahedron). To avoid redundant referencing of the microstates (which could be tolerated if corrected in the partition function), we also split the interstitial Voronoi cells. For example, consider a $\mathrm{T}$ site associated to a vacancy. It will be occupied only if the $\mathrm{H}$ is in the intersection of the Voronoï cell of the vacancy and of the T site. For the H case, it is 
(a)

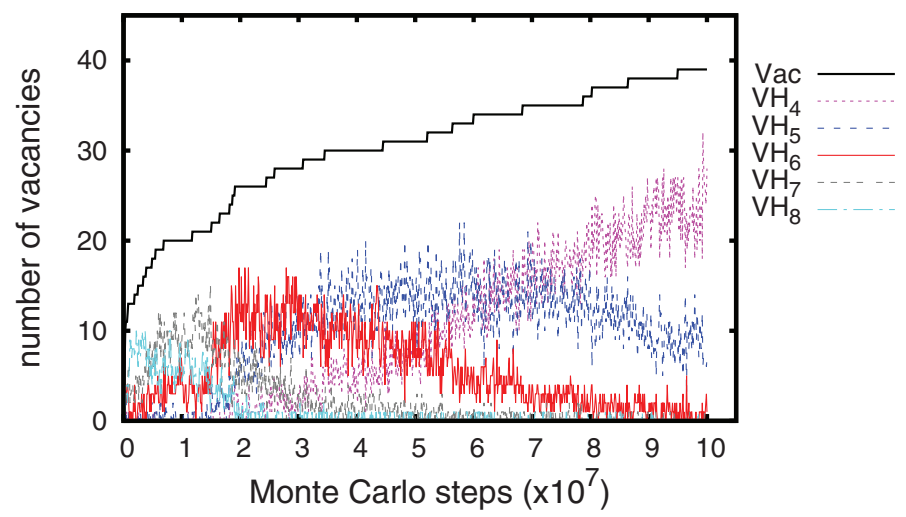

(b)

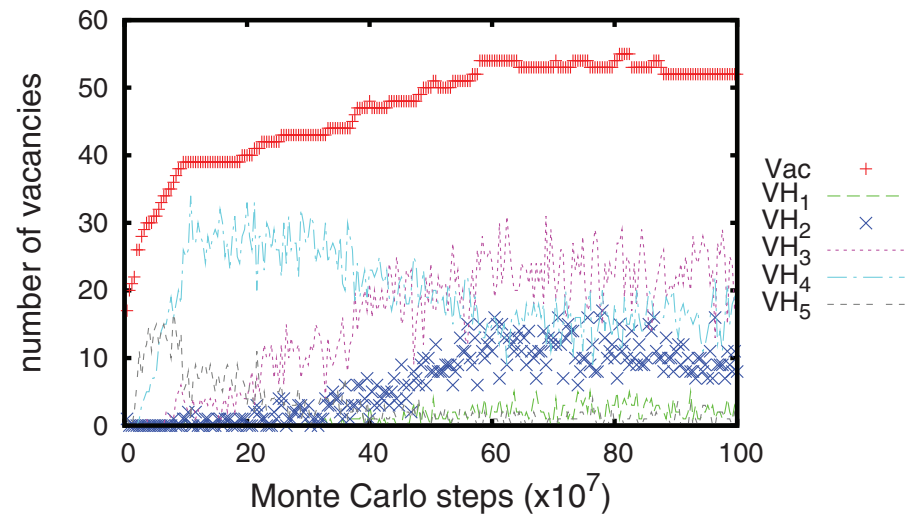

Fig. 2. Slow convergence of the total vacancy concentration and of the distribution of $V H_{n}$ clusters in a perfect $\mathrm{Al}$ crystal containing $1 \% \mathrm{H}$ at $\mathrm{T}=400 \mathrm{~K}$. The calculation involves:

displacements of $\mathrm{Al}$ and $\mathrm{H}$, insertion/deletions, volume changes, exchanges of $\mathrm{H}$ and cluster moves.

an efficient decomposition of space, because the $\mathrm{H}$ is physically relaxed towards the center of the vacancy. Defining a cluster is straightforward: it is the vacancy and the $\mathrm{H}$ belonging to the nodes it contains. Since, in the bulk, all the fcc sites are equivalent, the cluster move is reversible.

The Al-H system is used as a test system (Tanguy \& Mareschal (2005)) because of the low formation energy of the vacancies $(0.7 \mathrm{eV})$ and the large segregation energy $(-0.3 \mathrm{eV})$. To test the cluster move and obtain a large concentration of vacancies, the total (including trapped $\mathrm{H})$ concentration of hydrogen is $1 \%$. The reader is warned that the calculations were done with a potential which overestimated the vacancy - hydrogen binding: $-0.55 \mathrm{eV}$ instead of $-0.3 \mathrm{eV}$ as is given nowadays by ab initio calculations. The results are therefore only illustrative of the method (a new potential, the one used for intergranular segregation, shows that more than $1 \% \mathrm{H}$ is necessary to obtain large concentrations of vacancies at $400 \mathrm{~K})$. Figure 2 illustrates the slow convergence of the simulation. At the beginning of the simulation (Fig. 2a), the 
concentration of vacancies increases. Because of the $\mathrm{H}$ exchange moves, the newly created vacancies are highly multiply occupied (up to $8 \mathrm{H}$ per vacancies), but as more and more vacancies are created, the average occupancy decreases. Nevertheless, it is only when the vacancy concentration stabilizes and that the ordering of the vacancies is established that the true distribution of $\mathrm{H}$ is obtained (Fig. $2 \mathrm{~b}: \mathrm{VH}_{2}, \mathrm{VH}_{3}$ and $\mathrm{VH}_{4}$ are the dominant species). The presence of $\mathrm{VH}_{2}$ is really the signature of the ordering because it is when two vacancies are in first neighbor position that they can share $2 \mathrm{H}$ (Tanguy \& Mareschal (2005)).

This example highlights the importance of a good description of the ordering of the vacancies if the proper trapped hydrogen concentrations are to be measured.

\subsection{Hydrogen segregation to a grain boundary}

Are hydrogen-vacancy clusters really involved in the hydrogen damage of metals? If this is the case, the first place to look for these objects is where they can be formed in large quantities: at crack tips, in the dislocation core or at grain boundaries (GBs). Indeed, these defects are preferential sites for vacancy formation (low formation energy) and for $\mathrm{H}$ segregation. Let us first focus on the segregation of $\mathrm{H}$ alone. Lattice sites are defined in the core of the GB (Fig. 1). Keeping in mind that cluster moves are necessary when vacancies are present, we decided to keep the same number of interstitial sites (8T and 6O) per metal site as in the bulk. The interstitial sites are initially created at the same relative distance from the metal than in the bulk i.e. permutations of $(+/-0.25,+/-0.25,0)$ for the $\mathrm{T}$ site and $(+/-0.5,0,0)$ for the O site. But, because the fcc structure is disrupted by the presence of the GB, these sites are no longer sit on the borders of the Voronoï cells of the metal. This geometric construction leads a large number of different sites, many of them quite close from each other. The decomposition in Voronoï cells of the interstitial network becomes unnecessarily complex. To solve this problem, all the geometric sites that are closer than a radius $r_{i n t}=0.215 a_{0}$ are merged together on one site (the new position of the site is the average over the whole cloud of initial interstitial sites). After this procedure is done, a simple lattice, suitable for the definition of clusters, is obtained and used for $\mathrm{H}$ exchange moves and confined $\mathrm{H}$ displacements, just like in the bulk.

The reader who is not interested in details can skip this paragraph and the next one. The problem is that the interstitial sites are no longer equivalent, i.e. that their Voronoï cells don't have the same shape. So it is not always possible to perform the exchange and keep the same displacement. There are, at least, two solutions. First solution: the displacements are always taken at random in the new cell, which means that the ratio of the volume of the old and the new cell has to be taken into account in the metropolis criterion (this is the implementation that is used in Fig. 3). Second solution: it is easy to define the biggest sphere contained in all the Vornoï cells (just take half the smallest distance between two interstitial sites). Then, if an $\mathrm{H}$ particle is to be exchanged with a vacancy and its initial displacement is within this sphere (it is very often the case), then the displacement is conserved. Otherwise, a displacement is taken at random within the new Voronoï cell, but outside the sphere. Again, the ratio of the volumes (Voronoï volume - the sphere volume) must be included in the metropolis criterion. Figure $3 \mathrm{a}$ and $\mathrm{b}$ show a typical configuration obtained after equilibration with only displacement moves $(\mathrm{H}$ and $\mathrm{Al}$ ) and exchange moves for $\mathrm{H}$. The calculation is done at fixed volume and fixed total number of particles.

The next concern is about multiplicity of labelling of microstates. In the bulk, the interstitial sites are split between the different fcc Voronoï cells to have more possibilities for the $\mathrm{H}$ in the vacancies. This argument is still valid in the case of the intergranular sites. But this time, the interstitial Voronoï cells are not split, i.e. the same cell can contain several H. Of course, due to 


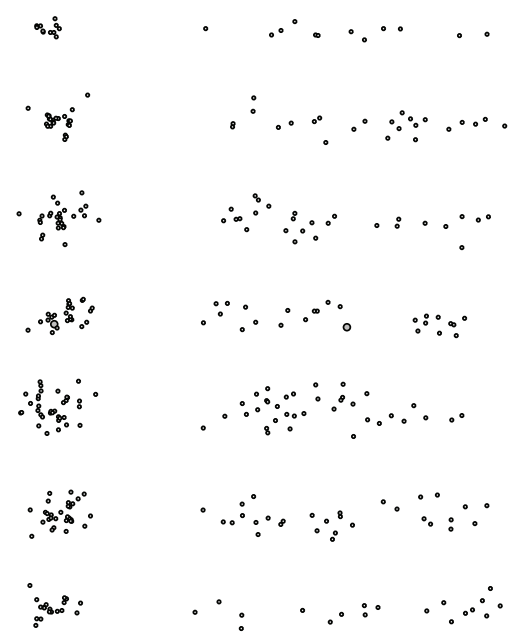

(a) (b)

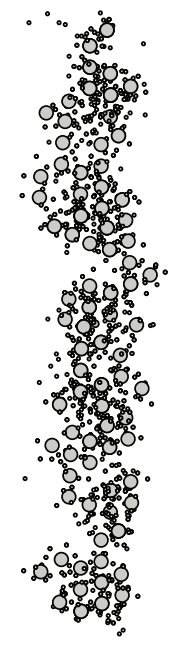

(c)

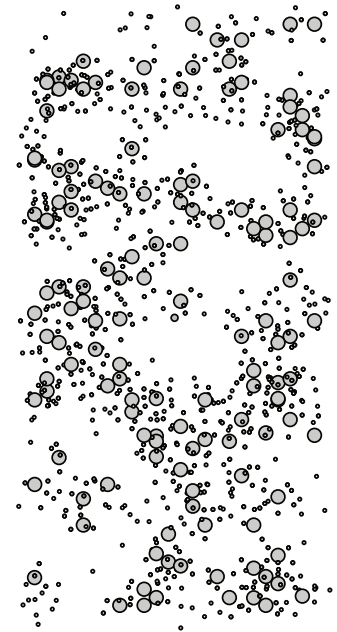

(d)

Fig. 3. A configuration taken from the Monte Carlo simulation of hydrogen segregation at the grain boundary shown on Fig. 1. (a) is a projection along the tilt axis of the grain boundary, like Fig. 1, (b) is a side view. The box contains $6 \mathrm{E}$ units which lead to this "ladder" structure: $\mathrm{H}$ segregates along the tilt axis in the $\mathrm{E}$ units. The results are obtained by Monte Carlo simulations with $\mathrm{H}$ exchanges and displacements of $\mathrm{Al}$ and $\mathrm{H}$ particles. The total number of $\mathrm{H}$ particles is fixed, no metal vacancies are allowed. The temperature is $400 \mathrm{~K}$ and the average bulk concentration is 300ppm atomic. The average grain boundary concentration is $15 \%$, i.e. $15 \mathrm{H}$ atoms are present for $100 \mathrm{Al}$ atoms in the region of half thickness $1 a_{0}$ around the grain boundary plane. (c) and (d) are similar views, but in the case where the total $\mathrm{H}$ content is increased and that vacancy creation is introduced.

$\mathrm{H}-\mathrm{H}$ repulsions, it never happens, unless a vacancy is present. At each step in the code, care is taken not to favor one representation of a state with respect to other ones. So, if no vacancies are present, and if we want to calculate the occupancy of one interstitial site, the sum should be made over all the different representations (Each physical T site, is represented by 4 different occupancies. All these should be summed to give the true occupancy of the site). In the case where some cells are multi-occupied (which could happen, in principle, only at very high $\mathrm{H}$ concentrations), the degeneracy of the labeling should be taken into account in the partition function. For the moment, we have not treated this case.

\subsection{Intergranular vacancy concentration in the presence of hydrogen}

When the Monte Carlo simulation is run with a large enough total content of $\mathrm{H}$ and low temperature, the segregation at the grain boundary is important. That is, in realistic conditions like $C_{H}=100 \mathrm{ppm}$ and $\mathrm{T}=300 \mathrm{~K}$, the local concentration is of the order of $3 \mathrm{H}$ for 10 metal atoms, which is large. If the concentration is increased, the level of $\mathrm{H}$ is large enough to disrupt the order in the boundary, as can be seen from the spreading of the peaks of the density profile perpendicular to the boundary. By doing so, the grain boundary tries to accommodate the large concentration by modifying its structure. This is a good case to test 
the possibility to form large concentrations of vacancies. A first run was made at $\mathrm{T}=400 \mathrm{~K}$ and $C_{H}>300 \mathrm{ppm}$, with insertion/deletions, cluster moves, exchanges and displacements. The acceptance rate is too low to reach convergence with this brute force approach. Nevertheless, it can be seen (Fig. $3 \mathrm{c}$ and d) that the grain boundary is enriched by a large number of vacancies. This confirms that superabundant vacancies should be present and well localized in the grain boundaries, since, in these temperature and concentration conditions they are not stable in the bulk (with this interaction energy $-0.3 \mathrm{eV}$ and formation energy of $0.7 \mathrm{eV}$ ). It is a strong indication that vacancies should be considered when large concentrations of solutes, in particular interstitial solutes, segregate. Technically, it is crucial to understand why the cluster moves have such a low acceptance rate in the grain boundary. To start with, we put aside the complex co-segregation of vacancies and solute interstitials and come back to the problem of simulating intergranular vacancies and especially their ordering at high concentration and low temperature.

\section{Hybrid Monte Carlo}

The strong relaxations induced in the grain boundary by the presence of vacancies severely reduce the efficiency of the vacancy exchange moves described above. In practice, if the simulation is started with all the vacancies in the bulk, the simple exchange moves can succeed in sampling the different configurations (i.e. the way the vacancies are distributed on the nodes), because the acceptance rate is not vanishingly small (see Fig. 2). On some GB sites, the relaxations bring the neighbors close to the node of the vacant site. Then, if the vacancy is exchanged with an atom, the relaxed neighbors and the new occupant overlap. The energy change becomes largely positive and the move is rejected. It becomes crucial when these sites are also those where the formation energy of the vacancy is the smallest, i.e. when the sites are the most occupied by vacancies. In this case it is impossible to get the proper concentrations and clustering of the vacancies.

Figure 4 quantifies this effect in the case of an exchange of a vacancy from one of such sites to another, geometrically equivalent one. A Monte Carlo simulation is run with exchange and displacement moves. The variation of the system energy during the exchange move is recorded. Its distribution is given by the curve labeled "simple X" (X stands for exchange). It is wide and centered around $7 \mathrm{eV}$. Note that no moves gave energy variations lower than $1 \mathrm{eV}$. It means that the acceptance rate, at low temperature, is vanishingly small. As a consequence, the system is trapped with vacancies occupying always the same sites. In particular, the geometrically equivalent sites are never visited. To solve the problem of the overlap, a first idea is to couple the exchange with displacement moves. Monte Carlo simulations are run first without vacancies and then with a single vacancy at a fixed location. The displacements of the particles are collected and the distributions are calculated for each node. They are usually gaussians, centered at, or close to, the node position (by construction of the lattice). Of course, the distributions are translated and distorted for the neighbors of the vacancy due to the large relaxations. A translation towards the vacancy is observed, but on a very limited number of sites. The idea is to measure the range of these relaxations and identify which neighbors are mostly affected. It defines a sphere of interaction around a vacancy (and, to insure reversibility, around the future vacancy node) where all the particles are attributed a new displacement after the exchange. Consider the initial vacancy, surrounded by relaxed neighbors. A particle is brought into the vacant site. A new displacement is taken according to the distribution measured without vacancies, for all the neighbors in the sphere (respectively for all the neighbors of the vacancy at its new location, according the distributions in the 


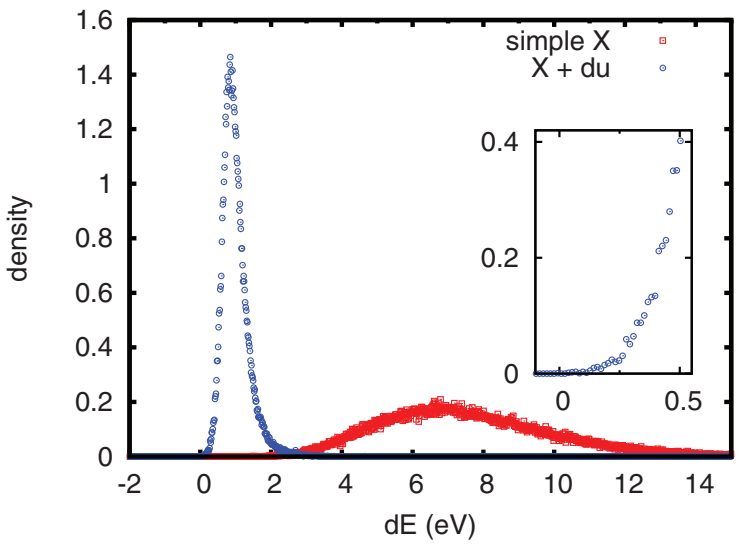

Fig. 4. Energy distribution for an exchange move between a vacancy with strong relaxations and a particle. Curve labeled "simple X" represents the variation of the energy obtained during the exchange alone, with the overlap between the relaxed neighbors and the new particle. Curve " $X+u$ " is obtained by combining the exchange with a displacement move which reduces the overlap. The inserted graph shows that moves with a low energy variation are rare (no moves with negative energy variation where found).

presence of vacancies). The metropolis criterion is modified to respect detailed balance. The new energy distribution for this "compound move" is shown on Fig. 4 (curve " $X+u^{\prime \prime}$ ). It is re-centered closer to the low energies, but still the probability that the energy variation is lower than $0.5 \mathrm{eV}$ is very small (see the inserted graph on Fig. 4). This was tested systematically by introducing more and more neighbors in the "interaction sphere", without success. The problem is that the new displacements are selected, at random, independently. There is no notion of collective arrangement of the neighbors.

There are several methods which bias the choice of the displacement of the atoms by taking into account the direction of the force and, usually, a random term (Allen \& Tildesley (1991)) is added: "smart Monte Carlo" (Rossky et al. (1978)), "dynamic Monte Carlo" (Kotelyanskii \& Suter (1992)), "force-bias Monte Carlo" (Cao \& Berne (1990))... The idea is to be guided by a "cheap" dynamics of the system to choose the trial positions. Faster convergence is achieved in the cases where concerted movements are necessary. In the problem described above, we want to make a large jump in configuration space by transporting, arbitrarily, the vacancy from one location to another. At the same time, we want to have both the initial and the final surroundings of the vacancy elastically relaxed and thermally exited (Uhlherr \& Theodorou (2006)) like they should be in equilibrium. In the next section, the exchange move is combined with Molecular Dynamics (MD) to obtain this effect.

\subsection{Algorithm}

Hybrid Monte Carlo (Duane et al. (1987)) (HMC) is a method which uses MD to generate a trial state and accepts it with the usual Metropolis rule. It has been adapted to condensed matter (Mehlig et al. (1992)) and applied in dense liquids (Desgranges \& Delhommelle (2008)), for example. It requires some modifications to be adapted to the vacancy problem that are detailed now. 
How can the exchange and the HMC moves be combined? The algorithm must be reversible and must satisfy detailed balance. The exchange alone is reversible. The HMC move is done by first, in the old state characterized by the occupancy of the nodes and the displacements of the particles (with some tolerance), taking random velocities for the particles according to the Maxwell distribution corresponding to the temperature $\mathrm{T}$. The integration of the motion is then performed with a time reversible, area preserving algorithm (the volume of phase space is preserved). For simplicity, the iso kinetic (Gauss) velocity verlet algorithm from Tuckerman is used (Tuckerman (2010)), so there is no need to consider the kinetic energy term in the energy balance. The new state is obtained after $2 \mathrm{nMD}$ integration steps. The reverse move is performed by selecting exactly the opposite velocities. The integration of $2 \mathrm{nMD}$ steps brings back the system to the original configuration.

If the MD steps are done after the exchange, the algorithm is not reversible. If one wants to use time reversibility, the exchange must be inserted in the middle of the MD trajectory. Then the compound move is:

- select velocities according to the Maxwell distribution

- perform nMD time reversible MD steps

- project the configuration on the lattice

- select a vacancy and a particle

- exchange them

- perform nMD time reversible MD steps

- project the configuration on the lattice

- calculate the energy variation and accept the new state according to the Metropolis rule

The "projection" is done by looping over the mobile particles and testing if they still belong to their original Voronoï cell. If not, the new Voronoï cell is found. If it is occupied by a particle, the move is not considered (not rejected, in the sense that it is not considered as a valid attempt). If it is occupied by a vacancy, the book keeping is made to exchange the occupancies of the sites and update the displacements.

Performing MD steps on the whole system is not necessary and prohibitively expensive. To speed up the calculations, only a limited number of particles are considered mobile. The compound move has two parameters: $\mathrm{nMD}$, the number of MD steps done before and after the exchange and a sphere radius $r_{M D}$ to define the mobile particles. Sometimes, a vacancy is brought by the exchange on a GB site where it is not stable. Then, even if the MD trajectory is short, the vacancy diffuses. This induces some practicle complications for choosing, reversibly, the vacancy and the particle for the exchange and calculate the probabilities that appear in the detailed balance.

\subsection{Detailed balance}

Let's take a closer look at the detailed balance condition in the case of a move from the bulk to the grain boundary. As mentioned earlier, it is not necessary to use the hybrid move for all the exchange moves, but only for some specific grain boundary sites. The sites can be decomposed in two categories: the "in" sites and the "out" sites. "in" means "in the region of the GB where the relaxations are particularly strong". The different moves can be in $\rightarrow$ out, in $\rightarrow$ in out $\rightarrow$ in and out $\rightarrow$ out. The out $\rightarrow$ out transition is done with simple exchanges. Let's focus on the out $\rightarrow$ in move. For the discussion of the detail balance, it is called the "direct" move and the inverse move is called the "return" move. 
(a)

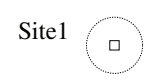

(b)

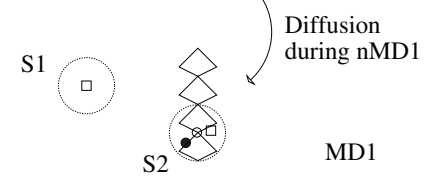

(c)

Exchange S1 \& S2

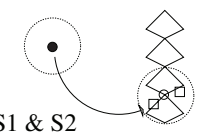

Diffusion during nMD2

(d)
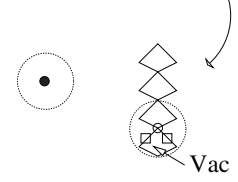

(h)
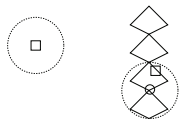

(g)

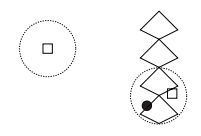

(f)

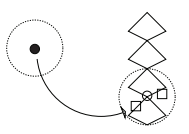

(e)

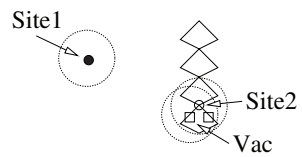

Fig. 5. Schematic representation of a hybrid Monte Carlo move bringing a bulk vacancy in the grain boundary (a to $d$ ) and the reverse move (e to $h$ ). The squares represent vacancies and the small circles, particles.

A vacant site is taken at random, with probability $1 / n V a c$, where $n V a c$ is the number of vacancies in the system. If the site is "out" (resp. "in") of the GB region, a trial move to bring the vacancy "in" (resp. "out") is constructed. Site1 is the vacant site selected. In the case of the out $\rightarrow$ in move it is in the bulk (Fig. 5a). Then another site is taken at random in the list of the "in" sites, with probability $1 / n$ GBsites (nGBsites is the number of sites considered "in"). This site is called Site2 (Fig. 5a). The list of mobile particles is constructed from the list of the nodes contained in two spheres of radius $r_{M D}$ around Site1 and Site2 (Fig. 5a). Then the velocities are taken randomly according to the Maxwell distribution, and nMD Molecular Dynamics steps are performed. Diffusion events occur during this relaxation, due to exchanges between mobile particles and vacancies (Fig. 5b). Note that, for preserving reversibility, if a mobile particle is exchanged with a vacancy not contained in the list of mobile sites, the move is abandoned. The particles are projected on the lattice, i.e. only one particle is affected to each Voronoï cell. If this is not possible, the move is abandoned. If the vacancy on Site1 diffuses, the move is abandoned because it is not possible to construct the return move. Otherwise, it is labeled S1. A site, labeled S2, is taken at random among the "in" sites contained in the list of mobile sites, with a probability $1 / n b G B m$ (m means "mobile"). The simple exchange move is performed between S1 and S2. nMD Molecular Dynamics steps are performed. The final configuration is projected on the lattice and the move is abandoned if it is not possible. A final check is performed on the vacancies which is necessary to guarantee reversibility. In particular, at the end of this procedure, at least one vacancy should be present in the sphere of radius $r_{M D}$ around Site2. It is labeled Vac (Fig. $5 \mathrm{~d}$ ). The motivation for this condition appears below, during the construction of the return move.

For the return, a way must be found to trace back Site1 and Site2, in the new configuration, and construct exactly the same list of mobile particles. A vacancy is taken at random. The 
probability to select Vac (Fig. 5e) is $1 / n V a c$. It is "in". An "in" site is taken at random in the sphere of radius $r_{M D}$ around Vac. The probability that the choice gives Site2 is $1 / \mathrm{nbGBm}$, where $n b G B m^{\prime}$ is the number of "in" sites in the sphere around Vac. This number has to be calculated at the end of the direct move (in d), because it enters detailed balance. But to be in the situation of doing such calculation, there must be at least one vacancy in the sphere around Site2 (that we called Vac by anticipation). For reversibility, this condition is imposed in the last step of the direct move (in d), as mentioned above.

An "out" site is taken at random. The probability to find Site 1 is $1 / n_{\text {out }}$, where $n_{\text {out }}$ is the number of sites not in the list of "in" sites. The mobile particles are created from the nodes included in two spheres of radius $r_{M D}$ around Site1 and Site2. They are the same than those of the direct move. The velocities are taken exactly as the opposite of the velocities at the end of the direct move, with the same probability as in the direct move. The trajectory is reversed during nMD steps. A vacancy is taken at random, among the "in" sites included in the list of mobile sites. The number of these vacancies is $n \operatorname{Vacm}$ ( $\mathrm{m}$ stands for "mobile"). The probability to choose the same vacancy as the one appearing after the exchange in the direct move is $1 / n V a c m$. This probability has to be evaluated during the direct move after the exchange. The exchange is performed. nMD steps are made to recover the initial configuration (a).

The condition imposing that a vacancy remains in the sphere around Site2, at the end of the direct move, is not a restrictive one because: fist, only mobile particles can diffuse, and therefore, the vacancy always stays in the mobile list; second, it is rare that the two spheres join. Another condition, is that no particles are allowed to leave from the mobile list, by an exchange with a vacancy on a node outside the mobile list. Otherwise, this particle will not be mobile during the inverse move.

The difficulty, when designing this algorithm, lies on the fact that the vacancies diffuse quite strongly during the relaxation. This is essentially because the exchanges force the occupancy of GB sites near the most favourable sites. A simple algorithm which abandons the move as soon as it detects that a vacancy leaves its original site during $M D$, gives a high abandon rate, of the order of $70 \%$. It is a large waste of computation time and also a waste of information on the system because these fast diffusion path are interesting to learn about the diffusion mechanism, in a statistical sense.

The "underlying matrix" $\alpha$ is not symmetric. Detailed balance is:

$$
\begin{aligned}
\frac{1}{\mathcal{Z}} \exp \left(-\beta H_{0}\right) \times \alpha_{\text {out } \rightarrow \text { in }} a c_{\text {out } \rightarrow \text { in }} & =\frac{1}{\mathcal{Z}} \exp \left(-\beta H_{n}\right) \times \alpha_{\text {in } \rightarrow \text { out }} a c c_{\text {in } \rightarrow \text { out }} \\
\alpha_{\text {out } \rightarrow \text { in }} & =1 / n V a c \times 1 / n G B \text { sites } \times 1 / n b G B m \\
\alpha_{\text {in } \rightarrow \text { out }} & =1 / n V a c \times 1 / n b G B m^{\prime} \times 1 / n_{\text {out }} \times 1 / n V a c m
\end{aligned}
$$

or

$$
\frac{a^{a c c_{\text {out } \rightarrow \text { in }}}}{\operatorname{acc}_{\text {in } \rightarrow \text { out }}}=\exp \left(-\beta\left(H_{n}-H_{0}-k T \ln \left(\alpha_{\text {in } \rightarrow \text { out }} / \alpha_{\text {out } \rightarrow \text { in }}\right)\right)\right)
$$

The metropolis criterion is:

- If $H_{n}-H_{o}-k T \ln \left(\alpha_{i n \rightarrow o u t} / \alpha_{\text {out } \rightarrow \text { in }}\right)<=0$ the move is accepted

- If $r=H_{n}-H_{o}-k T \ln \left(\alpha_{i n \rightarrow o u t} / \alpha_{\text {out } \rightarrow \text { in }}\right)>0$, a random number (rand) is taken between 0 and 1. If rand $<\exp (-\beta r)$, the move is accepted, otherwise it is rejected and the system stays in the old state (i.e. the same state is repeated twice in the Markov chain).

A similar algorithm is used for the in $\rightarrow$ out moves, and a simpler one for the in $\rightarrow$ in moves with a symmetric "underlying matrix". 


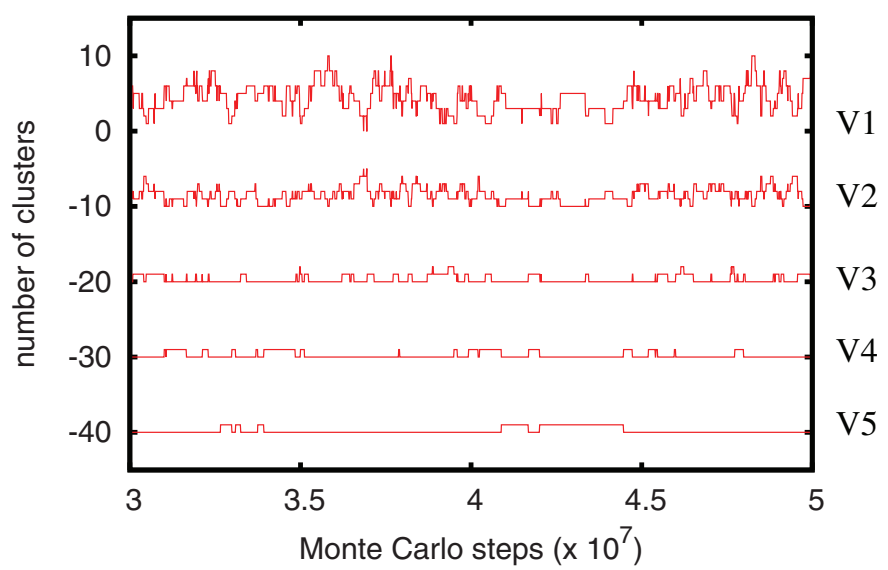

Fig. 6. Fluctuation of the number of vacancy clusters containing from 1 (V1) to 5 (V5) vacancies, as a function of the number of Monte Carlo steps. The total number of vacancies is 10 , the total number of accessible sites is 144 . Temperature is $200 \mathrm{~K}$. Each curve is translated down by -10 with respect to the previous one, for clarity.

\subsection{A first application to vacancies at a grain boundary}

This methodology is tested (Tanguy (n.d.)) by simulating the equilibrium of a fixed number of vacancies, in $\mathrm{Al}$, in a box containing the symmetrical tilt grain boundary $\Sigma 33(554)[110]$ (Vamvakopoulos \& Tanguy (2009)). The dimensions are such that three structural units (SU) (each one composed of two twin SU and two specific units of this family of tilt boundary, called E) are taken in the direction perpendicular to the tilt axis (Y) and $12 \mathrm{SU}$ along it ( $\mathrm{Z}$ direction). The calculation of the formation energy of the isolated vacancy shows (Vamvakopoulos \& Tanguy (2009)) that the E unit contains 2 atomic sites where this value is significantly lower than in the bulk. The presence of a vacancy in one of these sites induces large relaxations with all the problems of low acceptance rate for the exchanges discussed above. All these sites are gathered in a list, the list of "in" sites. The total number of "in" sites is 144. The total number of sites is of the order of 17000. Periodic boundary conditions are applied in the $\mathrm{Y}$ and $\mathrm{Z}$ directions, while rigid borders are imposed in the direction perpendicular to the interface (X direction). This is done to impose the lattice parameter of the perfect crystal at a reasonable distance from the interface.

The Monte Carlo simulation is composed of two types of moves:

- individual displacements of the particles in the whole system, as is commonly done to simulate the canonical ensemble

- compound hybrid Monte Carlo-exchange moves (HMC-X).

The proportions are 0.9995 displacements for 0.0005 HMC-X moves. Every 10000 microstep, a picture of the box, giving the location of each vacancy, is taken. Each of these images is post treated. At low temperature, the structure of the GB is such that the vacancies tend to align along the tilt axis, with some kinks. This ordering is roughly quantified by identifying the number of clusters of each size, from 1 to the total number of vacancies. A cluster is defined like a chain of first neighbors (it doesn't have to be a straight line). 
(a)

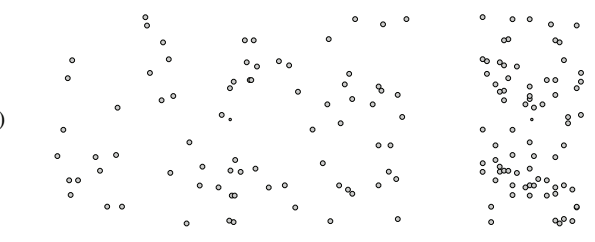

(b)

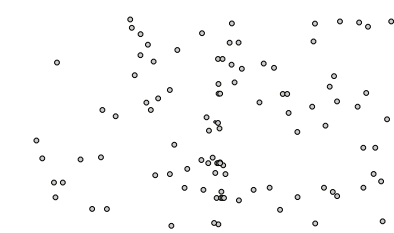

(c)

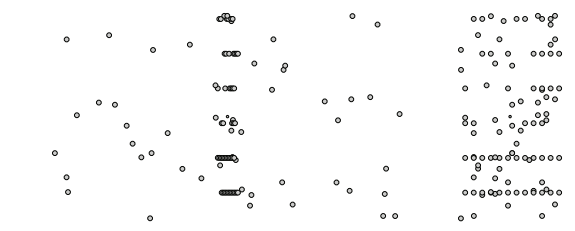

(d)

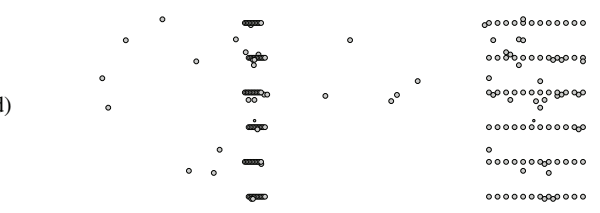

(e)

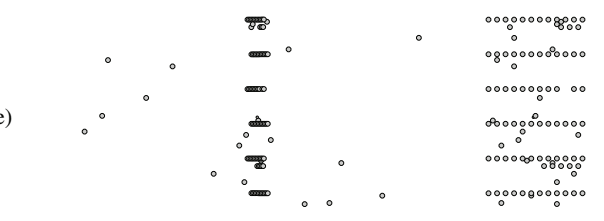

(f)

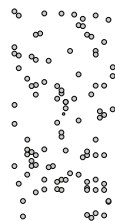

(g)

Fig. 7. A sequence of states, during equilibration of the system: (a) to (e) are front views (slightly tilted), (f) to (j) are the corresponding side views. The circles represent vacancies. The atoms are not shown.

Before trying to get some physical informations out of the simulations, it is important to evaluate its efficiency, i.e. to determine which range of thermodynamics variables (number of vacancies, temperature) that can be used as parameters for the Monte Carlo simulation and get a reasonable convergence, i.e. a meaningful approximate of the cluster distribution. Of course, this information should be obtained in a reasonable computer time (less than a month, on a single processor). A first test is made by taking 10 vacancies and distributing them 
among 144 "in" sites. Temperatures between 200K and 500K where used. Figure 6 shows the fluctuation of the number of clusters during the simulation (after some equilibration). It seems that a single long chain of vacancy is unstable with respect to shorter segments, even at the low temperature of $200 \mathrm{~K}$, in spite of a strong effective pair interaction $(0.3 \mathrm{eV}$ (Vamvakopoulos \& Tanguy (2009))). This graph and a visual control show that the system is not trapped and that the clusters are moved around the grain boundary as they should be when only short range order is established. The quantitative measure of the concentrations (Tanguy (n.d.)), as a function of temperature, still requires a check of the dependency on the number of MD steps in HMC-X and the radius of relaxation (these parameters where $n M D=200$ and $r_{M D}=1.6 a_{0}$, where $a_{0}$ is the lattice parameter of $\mathrm{Al}$ ). The method looks promising: the acceptance rate for HMC-X moves between geometrically equivalent sites is in between $30 \%$ and $40 \%$, with a weak temperature dependence. The drawback is its high computational cost, which means that a tuning should done to fix the proportions of regular exchange moves (without any relaxations) and HMC-X.

The last example (Fig. 7), is the equilibration, at $\mathrm{T}=200 \mathrm{~K}$, of a simulation containing 100 vacancies. An equal proportion of in $\rightarrow$ in and in $\rightarrow$ out moves are proposed. The vacancies are randomly distributed in the initial configuration (Fig. 7a and f) and gradually lead to the saturation of the lines, parallel to the tilt axis, composed of the sites where the formation energy is the lowest. Some more complex clusters are found that will be described later (Tanguy (n.d.)).

\section{Conclusion}

This paper shows the different steps in the development of a Monte Carlo simulation which gives, at the atomic scale, the equilibrium segregation of interstitial solutes and the vacancy concentration at pre-existing crystalline defects. The focus is on grain boundaries, but it can be adapted to dislocations. The flexibility in the design of the Monte Carlo moves, in particular unphysical moves like exchanges, cluster moves and the mixing with Molecular Dynamics, enables the sampling of a complex phase space of large dimensions and reveals details of the structure of the vacancy clusters that can not be guessed intuitively from the GB structure. Some work remains to be done to simulate the co-segregation of interstitial solutes and vacancies, with enough statistics to guarantee that the ordering the $V H_{n}$ clusters is properly sampled.

This work is supported by ANR blan2006 Hinter and blan2010 EcHyDNA.

\section{References}

Adams, D. J. (n.d.). Mol. Phys. 29: 307. .

Allen, M. P. \& Tildesley, D. J. (1991). Computer Simulation of Liquids, Oxford University Press, New York.

Bagchi, K., Andersen, H. C. \& Swope, W. C. (1996). Phys. Rev. Lett. 76: 255.

Bugeat, J. P., Chami, A. C. \& Ligeon, E. (1976). Phys. Rev. Lett. 58A: 127.

Cao, J. \& Berne, B. J. (1990). J. Chem. Phys. 92: 1980.

Dai, J., Kanter, J. M., Kapur, S. S., Seider, W. D. \& Sinno, T. (2005). Phys. Rev. B 72: 134102.

Denkowicz, M. J., Hoagland, R. G. \& Hirth, J. P. (2008). Phys. Rev. Lett. 100: 136102.

Desgranges, C. \& Delhommelle, J. (2008). Phys. Rev. B 77: 054201.

Duane, S., Kennedy, A. D., Pendleton, B. J. \& Roweth, D. (1987). Phys. Lett. B 195: 216.

Dünweg, B. \& Landau, D. P. (1993). Phys. Rev. B 48: 14182. 
Fukai, Y. (1993). The Metal Hydrogen System, Springer.

Fukai, Y. \& Ōkuma, N. (1994). Phys. Rev. Lett. 73: 1640.

Ganchenkova, M. G., Borodin, V. A. \& Nieminen, R. M. (2009). Phys. Rev. B 79: 134101.

Gunaydin, H., Barabash, S. V., Houk, K. N. \& Ozolinš, V. (2008). Phys. Rev. Lett. 101: 075901.

Henkelmann, G. \& Jónsson, H. (2001). J. Chem. Phys. 115: 9657.

Kotelyanskii, M. J. \& Suter, U. W. (1992). J. Chem. Phys. 96: 5383.

Landau, D. \& Binder, K. (2000). A Guide to Monte Carlo Simulation in Statistical Physics, Cambridge University Press.

Liu, Y.-L., Zhang, Y., Zhou, H.-B. \& Lu, G.-H. (2009). Phys. Rev. B 79: 172103.

Mehlig, B., Heermann, D. W. \& Forrest, B. M. (1992). Phys. Rev. B 45: 679.

Mezei, M. (n.d.). Mol. Phys. 40: 901.

Nørskov, J. K. \& Besenbacher, F. (1987). J. Less-Common Met. 130: 475.

Rossky, P. J., Doll, D. J. \& Friedman, H. L. (1978). J. Chem. Phys. 65: 4628.

Rowley, L. A., Nicholson, D. \& Parsonage, N. G. (n.d.). Mol. Phys. 31: 365.

Soisson, F., Barbu, A. \& Martin, G. (1996). acta mater. 44: 3789.

Sørensen, M. R., Mishin, Y. \& Voter, A. F. (2000). Phys. Rev. B 62: 3658.

Sørensen, M. R. \& Voter, A. F. (2000). J. Chem. Phys. 9599: 112.

Suzuki, A. \& Mishin, Y. (2005). J. Mater. Sci. 40: 3155.

Swope, W. C. \& Andersen, H. C. (1992). Phys. Rev. A 46: 4539.

Swope, W. C. \& Andersen, H. C. (1996). J. Chem. Phys. 102: 2851.

Tanguy, D. (n.d.). in preparation .

Tanguy, D. \& Mareschal, M. (2005). Phys. Rev. B 72: 174116.

Tateyama, Y. \& Ohno, T. (2003). Phys. Rev. B 174105: 67.

Tuckerman, M. (2010). Statistical Mechanics, Theory and Molecular Simulations, Oxford University Press, New York.

Uhlherr, A. \& Theodorou, D. N. (2006). J. Chem. Phys. 125: 084107.

Valleau, J. P. \& Cohen, L. K. (1980). J. Chem. Phys. 72: 5935.

Vamvakopoulos, E. \& Tanguy, D. (2009). Phys. Rev. B 79: 094116.

van Megen, W. \& Snook, I. (1980). J. Chem. Phys. 73: 4656.

Voter, A. F., Montalenti, F. \& Germann, T. C. (2001). Ann. Rev. Mater. Res. 32: 321.

Wang, J.-C., Pal, S. \& Fichthorn, K. A. (2001). Phys. Rev. B 63: 085403.

Wolverton, C., Ozoliņs̆, V. \& Asta, M. (2004). Phys. Rev. B 69: 144109. 


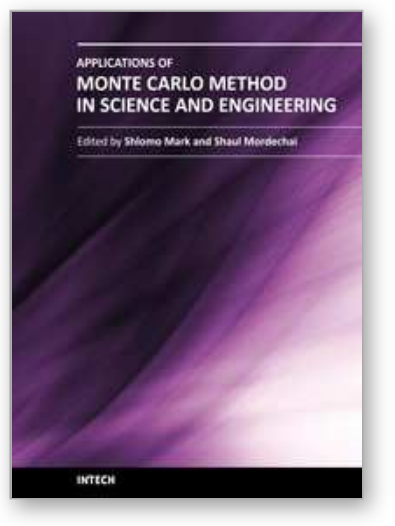

\section{Applications of Monte Carlo Method in Science and Engineering \\ Edited by Prof. Shaul Mordechai}

ISBN 978-953-307-691-1

Hard cover, 950 pages

Publisher InTech

Published online 28, February, 2011

Published in print edition February, 2011

In this book, Applications of Monte Carlo Method in Science and Engineering, we further expose the broad range of applications of Monte Carlo simulation in the fields of Quantum Physics, Statistical Physics, Reliability, Medical Physics, Polycrystalline Materials, Ising Model, Chemistry, Agriculture, Food Processing, X-ray Imaging, Electron Dynamics in Doped Semiconductors, Metallurgy, Remote Sensing and much more diverse topics. The book chapters included in this volume clearly reflect the current scientific importance of Monte Carlo techniques in various fields of research.

\section{How to reference}

In order to correctly reference this scholarly work, feel free to copy and paste the following:

Döme Tanguy (2011). Monte Carlo Methodology for Grand Canonical Simulations of Vacancies at Crystalline Defects, Applications of Monte Carlo Method in Science and Engineering, Prof. Shaul Mordechai (Ed.), ISBN: 978-953-307-691-1, InTech, Available from: http://www.intechopen.com/books/applications-of-monte-carlomethod-in-science-and-engineering/monte-carlo-methodology-for-grand-canonical-simulations-of-vacanciesat-crystalline-defects

\section{INTECH}

open science | open minds

\section{InTech Europe}

University Campus STeP Ri

Slavka Krautzeka 83/A

51000 Rijeka, Croatia

Phone: +385 (51) 770447

Fax: +385 (51) 686166

www.intechopen.com

\section{InTech China}

Unit 405, Office Block, Hotel Equatorial Shanghai

No.65, Yan An Road (West), Shanghai, 200040, China

中国上海市延安西路65号上海国际贵都大饭店办公楼 405 单元

Phone: +86-21-62489820

Fax: $+86-21-62489821$ 
(C) 2011 The Author(s). Licensee IntechOpen. This chapter is distributed under the terms of the Creative Commons Attribution-NonCommercialShareAlike-3.0 License, which permits use, distribution and reproduction for non-commercial purposes, provided the original is properly cited and derivative works building on this content are distributed under the same license. 